CBM 2018

\title{
Meshless Method for Simulation of Needle Insertion Into Soft Tissues: Preliminary Results
}

\author{
Adam Wittek ${ }^{1 *}$, George Bourantas ${ }^{1}$, Grand Roman, Joldes ${ }^{1}$, Anton Khau ${ }^{1}$, \\ Konstantinos Mountris ${ }^{2}$, Surya P. N. Singh ${ }^{3}$, Karol Miller ${ }^{1}$ \\ ${ }^{1}$ Intelligent Systems for Medicine Laboratory, \\ The University of Western Australia, \\ Crawley-Perth 6009, Western Australia, Australia \\ ${ }^{2}$ Aragón Institute of Engineering Research, IIS Aragón University of Zaragoza \\ ${ }^{3}$ School of Information Technology and Electrical Engineering \\ The University of Queensland (St Lucia Campus) \\ Brisbane, Queensland, Australia
}

\section{Introduction}

Needle insertion (placement) into human body organs is a frequently performed procedure in clinical practice [1]. Examples include placement of electrodes for deep brain stimulation [2], breast lesion biopsy [3], brachytherapy, and lumbar anaesthesia. Success of these procedures largely depends on the accuracy with which the needle tip reaches the anatomical target (the area of interest). The target position before surgery can be accurately determined from high-quality pre-operative medical images. However, the tissue deformation caused by interactions with the needle tends to change position of the target, which, in turn, can lead to inaccurate insertion [4]. If enough time is available, the target can be usually reached with assistance of image-guidance systems using ultrasound, computed tomography (CT) and magnetic resonance (MR) images [5,6]. However, such systems may not be sufficient to eliminate the need for multiply insertion attempts [5]. One possible way to decrease the risk of faulty needle placement can be to account for tissue deformations (and associated surgical target motion) when planning the needle insertion procedures. This can be achieved by employing computational biomechanics models to predict the tissue deformations during needle insertion. Consequently, needle insertion simulation has been a subject of substantial research effort with finite element algorithms as a method of choice for solving the equations of continuum mechanics governing the behaviour of body organs/tissues and their interactions with the needle [7-10]. However, ensuring stable and robust solution for large local strain caused 
by the needle and modelling of crack/discontinuity propagation due to the organ surface puncture and tissue penetration by the needle still remains a challenge.

In this study, for computing the tissue deformations due to needle insertion, we employ a meshless formulation of computational mechanics that relies on spatial discretisation in a form of a cloud of points. We use Meshless Total Lagrangian Explicit Dynamics (MTLED) framework previously developed and verified by our research group [11, 12]. Our previous research has indicated that the MTLED framework facilitates accurate prediction of the organ (brain) deformations due to surgery [13] and ensures robust and stable solution in a presence of very large strain $[11,14]$ and discontinuities/cracks induced by surgical dissection [15]. For modelling of interactions between the needle and soft tissues, we propose a kinematic approach that directly links deformation of the tissue adjacent to the needle with the needle motion. Unlike many previously proposed solutions, this approach does not require any assumptions about the exact mechanisms of such interactions.

We present the preliminary results of evaluation of the performance of the proposed method for simulation of needle insertion into soft tissues by comparing the modelling and experimental results obtained when conducting indentation and needle insertion into tissue phantom (cylindrical samples of soft incompressible Sylgard 527 silicone gel by Dow Corning) that exhibits the constitutive behaviour similar to the brain tissue. We acknowledge that the biofidelity of different materials in representing the brain tissue constitutive responses is a subject of extensive research [16]. Such research is, however, not within the scope of this study.

\section{Methods}

\subsection{Meshless Method for Simulation of Needle Insertion Into Soft Tissues}

\subsubsection{Meshless Total Lagrangian Explicit Dynamics Algorithm (MTLED) for Computing Soft Tissue Deformations}

We numerically solve the governing equations of continuum mechanics using the Meshless Total Lagrangian Explicit Dynamics (MTLED) framework that was introduced to compute deformation of soft tissues and other soft continua [11-13]. The MTLED is a Galerkin-type meshless method that uses an unstructured cloud of nodes to discretise the spatial domain (instead of elements like in Finite Element method) and spatial integration over the background grid of either hexahedral or tetrahedral integration cells. This way, 'good quality' mesh, that tends to be time 
consuming to build for continua with complex geometry (such as human body organs), is not needed. Placement of the nodes is automatic and their arrangement can be almost arbitrary. MTLED framework can handle large deformations and boundary changes [11]. As MTLED framework uses explicit time stepping, no iterations are needed even for strongly non-linear problems. More analytical and comprehensive description of the MTLED framework is available in papers [11-13] (and references therein).

For all models in this study, we used the MTLED framework with the Modified Moving Least Square (MMLS) shape functions [17] and boundary-conforming tetrahedral background integration cells with one integration (Gauss) point.

\subsubsection{A New Method for Modelling of Interactions Between the Needle and Soft Tissues Using Kinematic Approach}

For simulation of needle insertion into soft tissues, we propose a kinematic approach that does not require knowledge about the type of interactions between the needle and the tissue as it directly links the deformation of the tissues adjacent to the needle with the needle displacement/motion. As in most surgical procedures needle is much stiffer than the organ tissue, we consider the needle as a rigid body and represent it using a set of nodes that cannot move in relation to each other. Following the experimental literature on needle insertion into soft tissues [18], two phases of needle insertion are distinguished in our kinematic approach: i) Indentation where the organ surface deforms as a result of contact interactions with the needle tip; and ii) Tissue penetration by the needle that follows the puncture of the organ surface by the needle tip. During the insertion phase, the tissue is in contact with both the needle tip and the needle shaft.

(i) Indentation: During the indentation, only a small area on the organ surface (analysed continuum) is in contact with the needle. We represent this area by a subset of nodes located on the organ surface. During indentation, the displacement of these nodes equals the known (predefined) needle tip displacement. We monitor strain in the needle insertion area. When the strain exceeds a threshold value (referred to as puncture strain $\varepsilon_{p}$ ), the needle punctures the organ surface and the penetration phase starts.

(ii) Penetration: We define the nodes located in the support domain of the needle nodes. The nodes located in this support domain are then displaced by a fraction (referred to as of the deformation coefficient $C_{D}$ ) of the known (predefined) displacement of the needle. 
For software implementation of our method for simulation of needle insertion into soft tissues (soft continua), we used MATLAB version 2017b programming language (MathWorks https://au.mathworks.com/).

\subsection{Verification of the Proposed Meshless Method for Simulation of Needle Insertion Into Soft Tissues}

We follow the procedure outlined in Babuska and Oden [19] who recommend to verify new algorithms of computational mechanics against the established benchmark solutions. As the biomechanics research community has not formulated yet the benchmark solutions and established algorithms for needle insertion simulation, we conducted verification of our meshless method in the following two stages. In the first stage, we evaluated the accuracy and robustness of our meshless algorithm (MTLED) for computing soft continua deformations under large strain consistent with needle insertion. This was done by applying the MTLED framework to model the indentation of a cylindrical sample made from Sylgard 527 (Dow Corning, Midland, MI) silicone gel and comparing the forces predicted using MTLED framework with the results computed using established non-linear algorithms available in ABAQUS (version 16.14-1) finite element code (Dassault Systems, Simulia https://www.3ds.com/products-services/simulia/products/abaqus/) and the experimentally measured indentation force (Figure 1). The rationale for selecting Sylgard 527 gel was that this gel has been previously used in numerous studies as the brain tissue substitute $[20,21]$. As direct modelling of contact interactions requires calibration/tuning of the parameters of contact models, the indenter was not directly modelled. We prescribed the displacements of the nodes located on the sample surface that were in contact with the indenter face so that their displacement equalled the indenter displacement. As Sylgard 527 gel firmly stuck to the indenter face, there was no artefact related to the gel movement in relation to the indenter face (Figure 1).

In the second verification stage, we evaluated the performance of our kinematic approach for modelling the interactions between the needle and the tissue. This was done through application of this approach in modelling of needle insertion into a cylindrical sample of Sylgard 527 gel and comparing the results obtained from the model with the experimentally measured force acting on the needle (Figure 2).

All the experiments in this study were conducted using an in-house portable tension-compression test rig developed at the Intelligent Systems for Medicine Laboratory at The University of Western Australia [22] (see also http://isml.ecm.uwa.edu.au/ISML/) equipped with Burster 8532 EN load-cell (https://www.burster.com/) and MTS Temposonics CS H2 LVDT linear displacement sensor (http://www.mtssensors.com/) (Figure 1, Figure 2 and Figure 3). 
a)

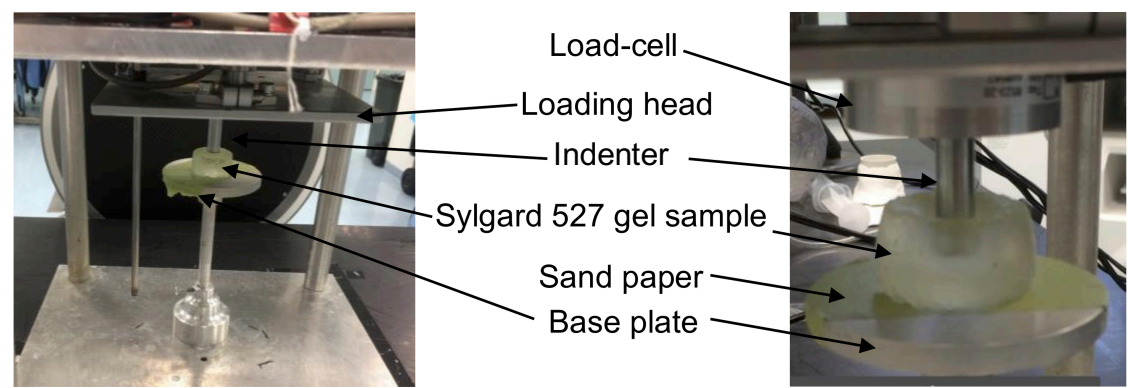

b)

c)

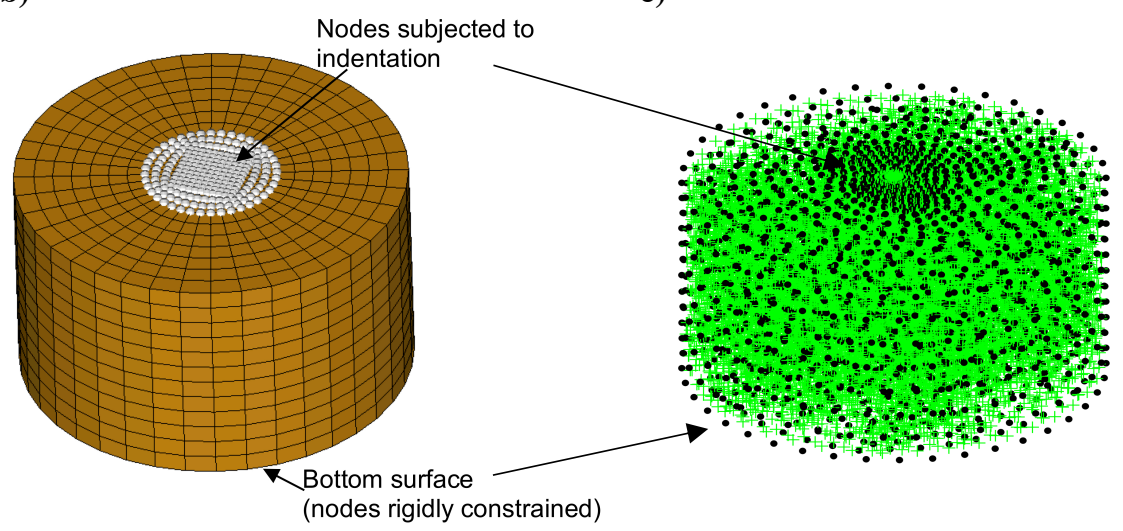

Figure 1 a) Indentation of cylindrical samples (diameter of $30 \mathrm{~mm}$, height of 17 $\mathrm{mm}$ ) of Sylgard 527 gel conducted in this study. The indenter (aluminium) diameter is $10 \mathrm{~mm}$. b) Finite element mesh for modelling of indentation of cylindrical samples of Sylgard 527 gel. The model was implemented using ABAQUS non-linear finite element code. The gel sample was discretised using 8,223 nodes and 6,000 eight-noded hexahedral elements with hybrid formulation (solid element C3D8H in ABAQUS finite element code). The hybrid formulation was used to prevent volumetric locking. c) Meshless discretisation (using the MTLED algorithm) for simulation of gel sample indentation. The sample was discretised using 8,233 nodes (black dots) and 44,343 background tetrahedral integration cells with one Gauss point (green crosses) per cell. 
a)

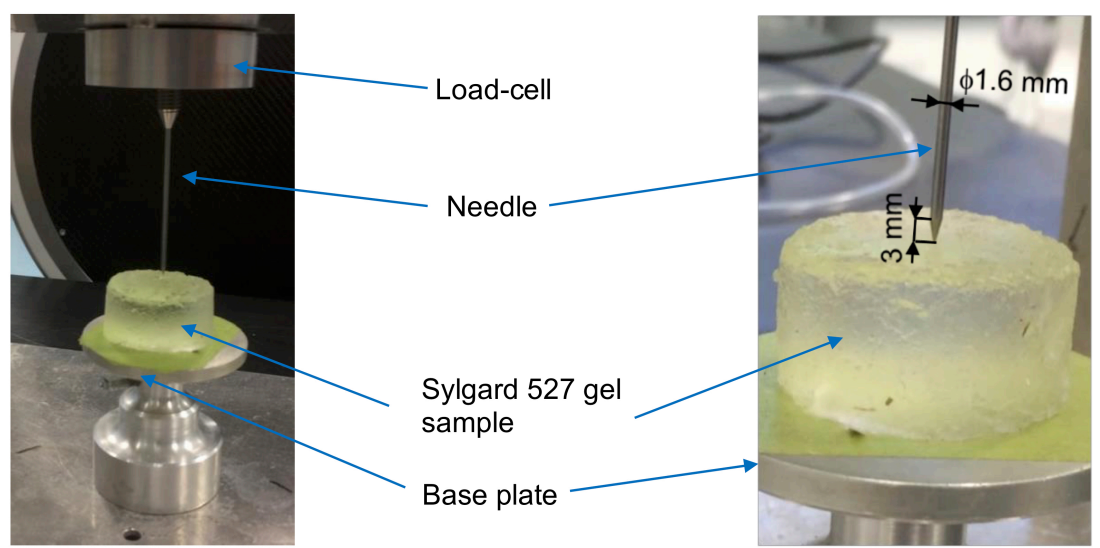

b)

Dense discretisation in the needle insertion area

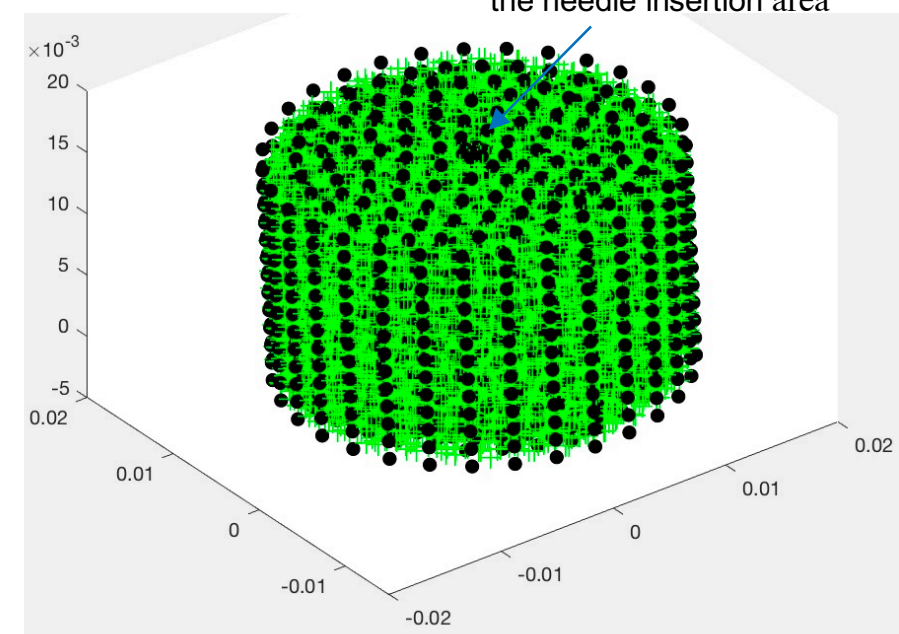

Figure 2 a) Needle insertion in the sample of Sylgard 527 gel. b) Meshless discretization (using the MTLED algorithm) for simulation of the experimental set-up shown in figure (a). The sample was discretised using 5,224 nodes (black dots) with 28,645 background tetrahedral integration cells with one Gauss point (green crosses) per cell. The coordinate frame indicates dimensions in meters. 


\subsubsection{Determining the Parameters for our Meshless Method for Simulation of Needle Insertion Into Soft Tissues}

Constitutive Model for Needle Insertion Simulation: The previous research has indicated that Sylgard 527 gel exhibits non-linear stress-strain relationship that can be accurately represented using hyperelastic constitutive models [21]. In this study, we used the neo-Hookean model [23]:

$$
W=\frac{\mu}{2}\left(I_{1}-3\right)+\frac{K}{2}(J-3)
$$

where $W$ is the strain energy, $\mu$ is the shear modulus, $K$ is the bulk modulus, $I_{1}$ is the first strain invariant of the right Cauchy Green deformation tensor, and $J$ is the volume ratio which equals the determinant of deformation gradient.

Data regarding the Sylgard 527 shear modulus have been previously reported in the literature $[16,21]$. However, as Sylgard 527 is a two-compound (part "A" and "B") gel, it's stiffness tends to exhibit sensitivity to even small variation in proportion of the two compounds and curing conditions. To determine the shear modulus for the specific compound proportion (nominally $1: 1$ weight ratio) and curing conditions (temperature of $60^{\circ} \mathrm{C}$ for 24 hours) used in this study, a cylindrical gel sample (height of $17 \mathrm{~mm}$ and diameter of $30 \mathrm{~mm}$ ) was subjected to compression (Figure 1). The bottom surface of the sample was placed on sandpaper glued to the rig base, i.e. this surface was rigidly constrained (Figure 3 ). As the loading plate was also covered with sandpaper, the sample's top surface was constrained in the horizontal plane and only vertical displacement was allowed. Following [21, 24], the experiments were modelled (the model is shown in Figure $3 b$ ) using the non-linear procedures available in ABAQUS finite element code. The shear modulus ( $\mu$ in Equation 1) was varied to obtain the computed force-displacement relationship close to the relationship determined from the experiments. [25, 26]. As Sylgard 527 is nearly incompressible, we used the Poisson's ratio of $0.49-$ a value used in our previous studies [11]. Using this protocol, we determined that the shear modulus of Sylgard 527 gel used to prepare the sample analysed in this study was $1678 \mathrm{~Pa}$. This result is consisted with the data we previously obtained [21] and with the literature [25, 26]. 
a)

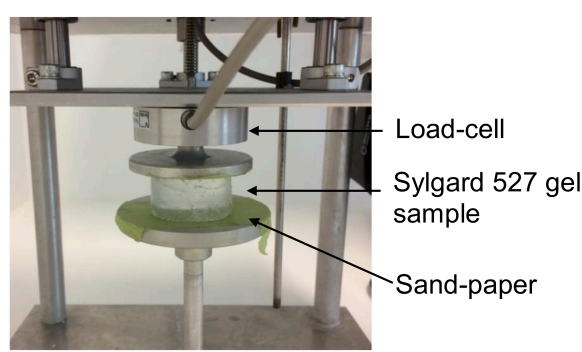

b)

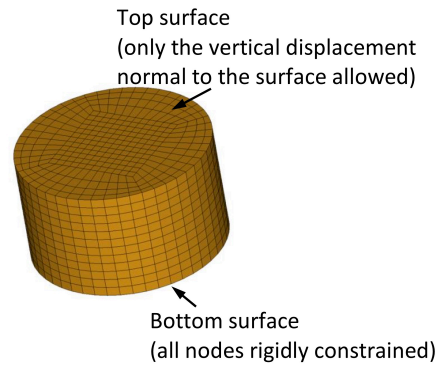

c)

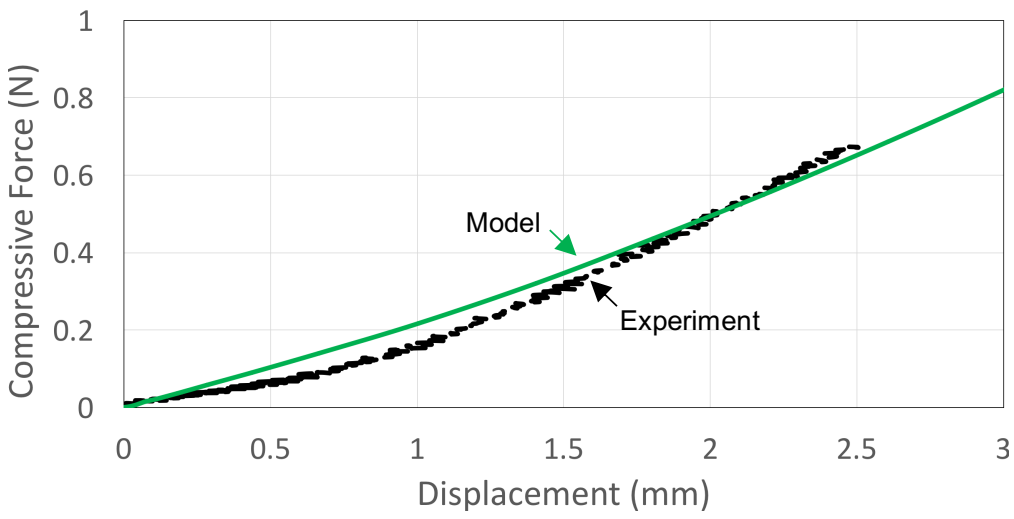

Figure 3 a) Compression of Sylgard 527 gel samples for determining the constitutive behaviour and shear modulus of the gel used in this study. b) Finite element model of the experiments using the set-up shown in figure (a). The model was implemented using ABAQUS non-linear finite element code. The gel sample was discretised using 4,032 eight-noded hexahedral elements with hybrid formulation (solid element $\mathrm{C} 3 \mathrm{D} 8 \mathrm{H}$ in ABAQUS finite element code). The hybrid formulation was used to prevent volumetric locking. c) Comparison of the results obtained using the model (green solid line) shown in figure (b) and the experimental data (black dotted line). The compression was done up to $2.5 \mathrm{~mm}$ ( $15 \%$ of the unloaded sample height).

\subsection{Determining the Parameters for New Algorithm for Modelling of Interactions Between the Needle and Soft Tissues Using Kinematic Approach}

As explained in Section 2.1.2, our algorithm for modelling of needle-tissue interactions uses two parameters: puncture strain $\varepsilon_{p}$ and deformation coefficient $C_{D}$ that links the deformation of the material adjacent to the needle with the needle 
displacement. These parameters were determined from the experiments using the needle insertion into the silicone gel samples (tissue phantom) (Figure 2).

Puncture strain $\varepsilon_{p}$ The needle force-insertion depth characteristics observed in this study exhibited a continuous increase without any visible local force drop that can be associated with the puncture (Figure 5). Consequently, the puncture strain was designated an arbitrarily low value of $\varepsilon_{p}=10^{-6}$. We considered also $\underline{\varepsilon}_{n}=0.0$, but decided against using it as it would not allow us to test the part of our new algorithm for modelling of interactions between the needle and tissue responsible for computing the puncture strain.

Deformation coefficient $C_{D}$ that links the deformation of the tissue adjacent to the needle with the needle motion Sylgard 527 gel tends to firmly stick/attach to smooth surfaces such as surgical needle shafts. From observation of the deformation the gel sample surface during the needle insertion, we observed that the gel adjacent to the needle moves/deforms by around $40-60 \%$ of the distance travelled by the needle tip. Consequently, we used the deformation coefficient of $C_{D}=0.4$.

\section{Results}

Verification of the MTLED framework performance for predicting the responses of soft incompressible continua was done through modelling of indentation of Sylgard 527 gel sample (for the experimental set up and its model, see Figure 1). We were able to obtain the reference solution using the established non-linear algorithms available in ABAQUS finite element code for the indentation depth of only $4.0 \mathrm{~mm}$. At around $4.1 \mathrm{~mm}$ the finite element solution started to diverge. We hypothesise that this was caused by distortion of the elements forming the finite element mesh in the indentation area. As the MTLED framework uses the computational grid in a form of a cloud points, it does not suffer from this type of instability. Therefore, using the MTLED framework, we obtained the stable result for the indentation depth of $10 \mathrm{~mm}$ which is the maximum indentation depth used in the experiments.

The force-indentation depth relationship from the MTLED framework is for practical purposes the same as the relationship obtained using non-linear finite element procedures available in ABAQUS finite element code and agree well with the experimental data (Figure 4). The difference between the modelling and experimental results for the indentation depth of $4 \mathrm{~mm}$ was only around $0.025 \mathrm{~N}$ which is around $5 \%$ of the measured force. The difference increases with the indentation depth. We propose the following two explanations for this phenomenon. The first is that we prescribed the displacements of the nodes on the sample surface in contact with the indenter face and did not directly model the indenter. This does not account for the contact between the gel and cylindrical surface of the indenter which occurs when the indentation depth increases (see Figure 1a). The second possible explanation for the fact that the differences between the modelling and experimental result increased with the indentation depth can be that the neo-Hookean hyperelastic 
constitutive model we use here may not accurately represent Sylgard 527 stressstrain relationship for large strain. In this model, the strain energy is proportional to the second power of the principal stretch ratios (first left deformation tensor invariant) (see Equation 1). More complex hyperelastic models that include higher order terms in strain energy function (such as Ogden hyperelastic model used in our previous studies [20,23]) may be needed to accurately represent the Sylgard 527 gel constitutive behaviour for large strain.

a)

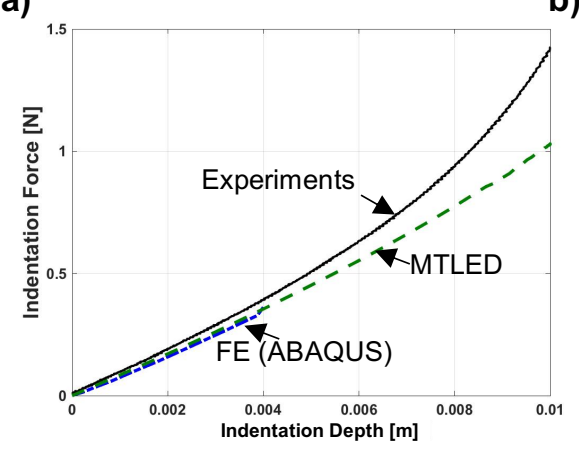

b)

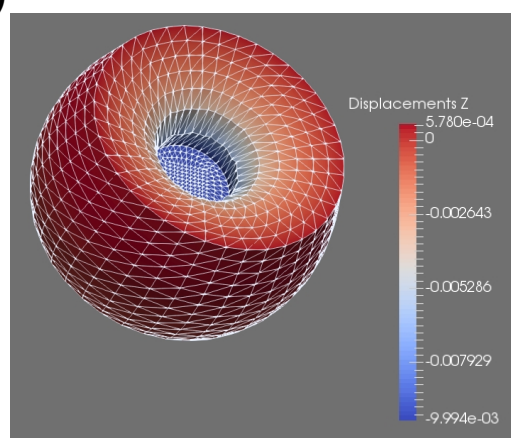

Figure 4 a) Verification of the Meshless Total Lagrangian Explicit Dynamics (MTLED) framework through modelling of the experiments (indentation of Sylgard 527 gel samples) conducted using the set-up shown in Figure 1. Comparison of the force - indentation depth relationship obtained using the MTLED framework (green dotted line), non-linear finite element procedures available in ABAQUS finite element (FE) code (blue dotted line), and the experimental data (black solid line) average from three experiments. Using ABAQUS finite element code we were able to obtain the results for the indentation depth of up to only $4 \mathrm{~mm}$ after which the solution diverged. For the indentation depth of up to $4 \mathrm{~mm}$, the results obtained using the MTLED framework and ABAQUS code are very close and cannot be visually distinguished. b) Overall deformation of the meshless model for the indentation depth of $10 \mathrm{~mm}$ (the displacement scale in the figure is in meters). The interpolating nodes were connected to form the triangles to visualise the deformed model surface. The meshless discretisation is shown in Figure 1c.

The results of modelling of needle insertion into a cylindrical sample of Sylgard 527 gel indicate that our kinematics approach for representing the needle-tissue (soft tissue phantom) interactions implemented in the MTLED framework not only provides a stable solution for large local strain induced by the needle (Figure 5), but also accurately predicts the general behaviour and magnitude of the needle force-insertion depth relationship (Figure 6). The maximum difference between the force predicted by our method and experimental results was only around 
$25 \%$ (under $0.01 \mathrm{~N}$ ). This difference can be minimised by adjusting the parameter $C_{D}$ that links the deformation of the material adjacent to the needle with the needle displacement. However, this fairly straightforward exercise has not been conducted here as we are more interested in assessing predictive rather than explanatory power of our approach.

a)

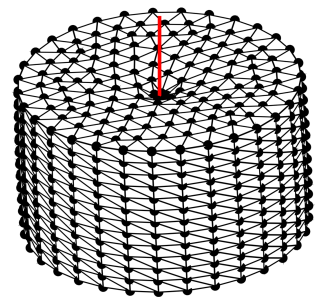

b)

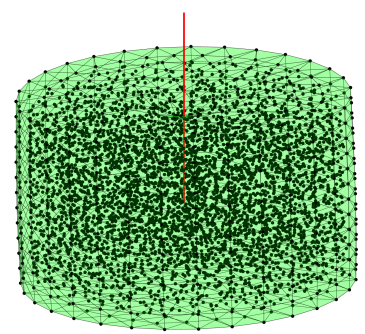

Figure 5 Modelling of needle insertion into the Sylgard 527 gel sample using our meshless method for needle insertion simulation. a) The predicted shape of the sample surface for the insertion depth of $10 \mathrm{~mm}$. The interpolating nodes are connected to form the triangles to visualise the deformed model surface.) b) Position of the interpolating nodes for the needle insertion depth of $10 \mathrm{~mm}$.

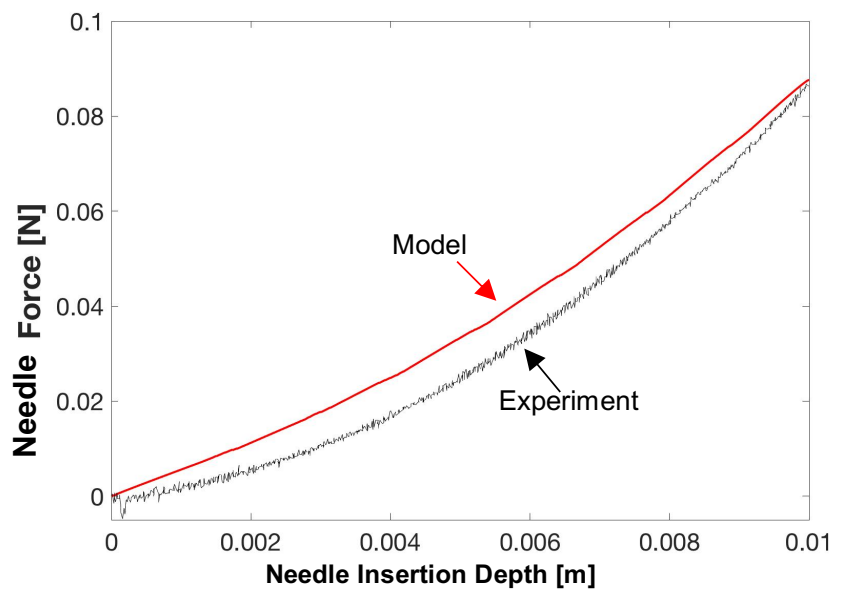

Figure 6 Comparison of the force - insertion depth relationship when inserting a needle into the cylindrical samples of Sylgard 527 gel obtained using our meshless method for needle insertion simulation (red line) and the experimental data (black line). The needle insertion was conducted for the depth of up to $10 \mathrm{~mm}$. The experimental set-up is shown in Figure 2. The model is shown in Figure 2 and Figure 5. 


\section{Discussion}

The results of modelling of indentation of tissue phantom (Sylgard 527 gel sample) confirm the capability of Meshless Total Explicit Lagrangian (MTLED) framework, in providing the stable solution of equations of continuum mechanics for large localised deformations/strain where the procedures available in established non-linear finite element codes (we used the ABAQUS code) fail (Figure 4). This makes the MTLED framework a suitable method for simulation of needle insertion and other surgical procedures.

The results of modelling of needle insertion into a Sylgard 527 gel sample indicate that our kinematics approach for representing the needle-tissue (soft tissue phantom) interactions, in which the deformation of the material adjacent to the needle is directly linked with the needle motion without any assumptions regarding the exact mechanism of the interactions between the needle and tissue (soft tissue phantom), correctly predicts the general behaviour of the needle force-insertion depth relationship (Figure 6). The predicted force magnitude differed by only $25 \%$ from the experimentally observed value (Figure 6). Similar difference (20\%) between the modelling and experimental results was obtained by Oldfield et al. [16] who used extensive calibration of their high-resolution cohesive element model of needle insertion into the gelatine phantom. It might be expected that with more elaborate calibration, that includes determining the deformation coefficient $C_{D}$ (that links the deformation of the material adjacent to the needle with the needle motion) by tracking the deformations at the selected locations within the tissue sample (tissue phantom) in the needle insertion area, the accuracy of force prediction obtained using our kinematics approach for modelling of interactions between the needle and tissue (tissue phantom) may improve. Another possible reason for the differences between the results of needle insertion simulation and experimental data observed here can be that, as discussed when analysing the results of modelling of Sylgard 527 gel sample indentation, the neo-Hookean hyperelastic constitutive model we use here may not accurately represent Sylgard 527 stress-strain relationship for large strain. This can be addressed through application of more complex hyperelastic models that include higher order terms in strain energy function (such as Ogden hyperelastic model).

The needle force-insertion depth characteristics obtained in this study using tissue phantom (Sylgard 527 gel) do not exhibit any significant force decrease/drop we observed in our previous experiments on needle insertion into swine brain specimens [24]. Consequently, we were not able to evaluate the performance of our meshless method for simulation of needle insertion into soft tissues for this important aspect of the needle force - insertion depth behaviour. Such evaluation requires studies that would compare the modelling results against the experimental data obtained using tissue/organ specimens rather than tissue phantoms. 


\section{Acknowledgements}

This research was supported by the Australian Government through the Australian Research Council's Discovery Projects funding scheme (project DP160100714). Adam Wittek and Anton Khau thank Dr Barry Doyle for use of the VascLab tension-compression rig.

\section{References}

1. Zhu, Y., Magee, D., Ratnalingam, R., Kessel, D.: A Training System for Ultrasound-Guided Needle Insertion Procedures. pp. 566-574. Springer Berlin Heidelberg (2017)

2. Vega, R.A., Holloway, K.L., Larson, P.S.: Image-guided deep brain stimulation. Neurosurg Clin N Am 25, 159-172 (2014)

3. Apesteguía, L., Pina, L.J.: Ultrasound-guided core-needle biopsy of breast lesions. Insights into Imaging 2, 493-500 (2011)

4. Leibinger, A., Oldfield, M.J., Rodriguez y Baena, F.: Minimally disruptive needle insertion: a biologically inspired solution. Interface Focus 6, (2016)

5. Fichtinger, G., Deguet, A., Masamune, K., Balogh, E., Fischer, G.S., Mathieu, H., Taylor, R.H., Zinreich, S.J., Fayad, L.M.: Image overlay guidance for needle insertion in CT scanner. IEEE Transactions on Biomedical Engineering 52, 1415-1424 (2005)

6. Ungi, T., Beiko, D., Fuoco, M., King, F., Holden, M.S., Fichtinger, G., Siemens, D.R.: Tracked ultrasound snapshots enhance needle guidance for percutaneous renal access: A pilot study. Journal of Endourology (2014)

7. Bui, H.P., Tomar, S., Courtecuisse, H., Cotin, S., Bordas, S.P.A.: Real-Time Error Control for Surgical Simulation. IEEE Trans Biomed Eng 65, 596-607 (2018)

8. Courtecuisse, H., Allard, J., Kerfriden, P., Bordas, S.P.A., Cotin, S., Duriez, C.: Real-time simulation of contact and cutting of heterogeneous soft-tissues. Medical Image Analysis 18, 394-410 (2014)

9. DiMaio, S.P., Salcudean, S.E.: Needle insertion modeling and simulation. IEEE Transactions on Robotics and Automation 19, 864-875 (2003)

10. Oldfield, M., Dini, D., Giordano, G., Rodriguez y Baena, F.: Detailed finite element modelling of deep needle insertions into a soft tissue phantom using a cohesive approach. Computer Methods in Biomechanics and Biomedical Engineering 16, 530-543 (2013)

11. Horton, A., Wittek, A., Joldes, G.R., Miller, K.: A meshless Total Lagrangian explicit dynamics algorithm for surgical simulation. International Journal for Numerical Methods in Biomedical Engineering 26, 977-998 (2010) 
12. Joldes, G.R., Chowdhury, H., Wittek, A., Miller, K.: A new method for essential boundary conditions imposition in explicit meshless methods. Engineering Analysis with Boundary Elements 80, 94-104 (2017)

13. Miller, K., Horton, A., Joldes, G.R., Wittek, A.: Beyond finite elements: A comprehensive, patient-specific neurosurgical simulation utilizing a meshless method. Journal of Biomechanics 45, 2698 - 2701 (2012)

14. Kang, A.: Using Meshless Method to Predict Brain Phantom Deformation Upon Needle Insertion. 36 pages. Intelligent Systems for Medicine Laboratory, Master of Professional Engineering Thesis; Intelligent Systems for Medicine Laboratory, The University of Western Australia, CrawleyPerth, Western Australia (2016)

15. Jin, X., Joldes, G.R., Miller, K., Yang, K.H., Wittek, A.: Meshless algorithm for soft tissue cutting in surgical simulation. Computer Methods in Biomechanics and Biomedical Engineering 17, 800-817 (2014)

16. Leibinger, A., Forte, A.E., Tan, Z., Oldfield, M.J., Beyrau, F., Dini, D., Rodriguez y Baena, F.: Soft tissue phantoms for realistic needle insertion: A comparative study. Ann Biomed Eng 44, 2442-2452 (2016)

17. Chowdhury, H.A., Wittek, A., Miller, K., Joldes, G.R.: An element free Galerkin method based on the Modified Moving Least Squares approximation. J Sci Comput 71, 1197-1211 (2017)

18. Washio, T., Chinzei, K.: Needle force sensor, robust and sensitive detection of the instant of needle puncture. In: 7th International Conference on Medical Image Computing and Computer-Assisted Intervention MICCAI 2004, Proceedings: Lecture Notes in Computer Science 3217, pp. 113-120 (2004)

19. Babuska, I., Oden, J.T.: Verification and validation in computational engineering and science: basic concepts. Computer Methods in Applied Mechanics and Engineering 193, 4057-4066 (2004)

20. Bottan, S., Poulikakos, D., Kurtcuoglu, V.: Phantom Model of Physiologic Intracranial Pressure and Cerebrospinal Fluid Dynamics. IEEE Transactions on Biomedical Engineering 59, 1532-1538 (2012)

21. Ma, J., Wittek, A., Singh, S., Joldes, G., Washio, T., Chinzei, K., Miller, K.: Evaluation of accuracy of non-linear finite element computations for surgical simulation: study using brain phantom. Computer Methods in Biomechanics and Biomedical Engineering 13, 783 - 794 (2010)

22. Agrawal, S., Wittek, A., Joldes, G., Bunt, S., Miller, K.: Mechanical Properties of Brain-Skull Interface in Compression. In: Doyle, B., Miller, K., Wittek, A., Nielsen, M.F.P. (eds.) Computational Biomechanics for Medicine X: New Approaches and New Applications, 83-91. Springer International Publishing, Cham (2015)

23. Rivlin, R.S., Sawyers, K.N.: The strain-energy function for elastomers. Transactions of the Society of Rheology 20, 545-557 (1976) 
24. Wittek, A., Dutta-Roy, T., Taylor, Z., Horton, A., Washio, T., Chinzei, K., Miller, K.: Subject-specific non-linear biomechanical model of needle insertion into brain. Computer Methods in Biomechanics and Biomedical Engineering 11, 135-146 (2008)

25. Ivarsson, J., Viano, D.C., Lövsund, P., Aldman, B.: Strain relief from the cerebral ventricles during head impact: experimental studies on natural protection of the brain. Journal of Biomechanics 33, 181-189 (2000)

26. Basati, S.S., Harris, T.J., Linninger, A.A.: Dynamic Brain Phantom for Intracranial Volume Measurements. IEEE Transactions on Biomedical Engineering 58, 1450-1455 (2011) 